\title{
Restrictions and opportunities for UC cannabis research
}

\author{
Cannabis is legal in California but illegal in the United States. The plant's ambiguous status cuts off \\ many avenues of cannabis research — but leaves other approaches wide open.
}

Though researchers who wish to study the cannabis plant face strict federal constraints, opportunities to conduct cannabis research are not scarce around the UC system.
W hen California voters approved Proposition 64 in 2016, legalizing recreational cannabis for adults, they fundamentally altered the state's cannabis landscape. They also, albeit unintentionally, furnished UC researchers with intriguing new avenues of potential inquiry - many of which are blocked by federal law and pursuant UC policy. For example, researchers interested in the cannabis-derived sprays and beverages readily available at California's retail cannabis establishments cannot obtain those products for research purposes by any permissible means. Licensed cannabis businesses dot the state today, but cannabis research still operates within the same strict constraints that have hindered it since legalization was a futile sentiment on a bumper sticker.

Because state law is subordinate to federal law, Proposition 64 is subordinate to the 1970 Controlled Substances Act. Associated with that act is a "scheduling" apparatus, overseen by the Drug Enforcement Administration (DEA), that identifies cannabis as ripe for abuse and devoid of medical merit. Thus, along with heroin and other Schedule I substances, the

Online: https://doi.org/10.3733/ca.2019a0023 psychoactive variety of cannabis cannot under federal law be cultivated, processed, sold, consumed - or, for the most part, researched. (However, change is afoot for research on industrial hemp, the non-psychoactive variety of cannabis - more on this below.)

The University of California, as a law-abiding institution, complies with the Controlled Substances Act and its nearly total cannabis prohibition. As an institution that receives federal funding, UC complies with the Drug-Free Workplace Act and the Safe and DrugFree Schools and Communities Act - which require universities, if they wish to receive federal funding, to implement policies prohibiting on-campus activities such as possession or use of controlled substances. UC personnel, including staff, faculty and UC Cooperative Extension (UCCE) specialists and advisors, are therefore prohibited, in their professional capacities, from direct contact with the cannabis plant or its extracts, and also from certain types of indirect contact. They cannot, for example, visit cannabis cultivation sites or advise cannabis growers on topics such as yield increases. Researchers can't even use cannabis or cannabis-derived products in medical studies - unless they fulfill a rather daunting set of federal (and state) requirements. 
Those requirements for medical studies include obtaining a Schedule I license from the DEA; submitting research protocols for Food and Drug Administration (FDA) approval; submitting to the FDA an investigational new drug application (if human subjects are involved in the research); and, as a non-federal matter, gaining the approval of a state entity, the Research Advisory Panel of California (housed within the Office of the Attorney General). If all goes well, researchers can then obtain cannabis or cannabis-derived substances from a DEA-licensed cultivator, a DEAregistered bulk manufacturer or, with a DEA import license, a foreign exporter. The only DEA-licensed cannabis cultivator is the University of Mississippi, which grows the plant under a contract funded by the National Institute on Drug Abuse (an entity within the Department of Health and Human Services). Bulk manufacturers of cannabis products such as tetrahydrocannabinol (THC) - the psychoactive component in cannabis - include, for example, the Massachusettsbased life science company MilliporeSigma (until recently, Sigma-Aldrich). Providers of imported cannabis products - such as Tilray, a Canadian firm - must be based in jurisdictions where such products are legal.

No matter which path researchers choose, the process isn't fast or easy. "You need a patient, dedicated team willing to jump through extra hoops at the institutional, state and federal levels," says Jeffrey Chen, Executive Director of UCLA's Cannabis Research Initiative. Even so, Chen reports, federal restrictions on types and sources of cannabis products can prevent researchers from conducting cannabis studies at all. And again, only medical researchers are eligible to obtain cannabis for research. Those who wish to perform agronomic studies, for example, are simply out of luck.

For all that, opportunities to research cannabis are not scarce around the UC system. Observational studies of cannabis users are permissible, though the cannabis in question cannot be provided by the university and must be consumed off campus. Researchers interested in the legal or economic dimensions of cannabis, or in cannabis policy, will discover few obstacles in the Controlled Substances Act. Several UC researchers are vigorously investigating the environmental consequences of cannabis cultivation - and in fact Proposition 64 has effectively expanded the scope for such research. According to Ted Grantham, a UCCE specialist at UC Berkeley and co-director of the UCB Cannabis Research Center, researchers can now interact with cannabis growers - to learn, for example, about their cultivation practices - in a way that grower reluctance previously precluded. Today, Grantham reports, "a subset of growers is very interested in daylighting the cannabis industry to establish its legitimacy as an agricultural crop rather than an illicit substance."

In years to come, UC investigators will likely perform extensive research on industrial hemp. This form

\section{Cannabis and hemp — what's the difference?}

- Cannabis and industrial hemp are precisely the same plant —Cannabis sativa - but they differ in their concentration of the psychoactive compound tetrahydrocannabinol (THC).

Under the stipulations of the 2018 Farm Bill, the THC content of industrial hemp may not exceed $0.3 \%$ (on a dry-weight basis). Cannabis sativa whose THC content exceeds that level is regarded as marijuana by the federal government. (The state of California prefers the scientific term "cannabis" to the racially charged term "marijuana.")

- Psychoactive cannabis is mainly used to produce a "high" — though many California consumers use cannabis for medicinal purposes as well. Hemp is used for fiber and in diverse industrial applications, and also as a source of cannabidiol (CBD, a non-psychoactive compound purported to confer numerous health benefits).

In the past, hemp was bred mainly for fiber and cannabis was bred to produce large buds that were rich in THC. The appearance of hemp and cannabis plants therefore usually differed. Today, because hemp is often bred to produce CBD - concentrated, like THC, in the buds of Cannabis sativa industrial hemp and psychoactive cannabis often differ little in appearance.

of cannabis, which contains extremely small amounts of THC, is useless for producing a "high" - but very useful for making fabrics, insulation, paper and more. Until recently, however, federal law did not distinguish between low-THC hemp and high-THC cannabis - nor between THC and cannabidiol (CBD), a nonpsychoactive cannabis compound purported to relieve medical conditions ranging from arthritis to anxiety.

The legal landscape for hemp and CBD began to change on the federal level in 2014, when that year's Farm Bill allowed universities to cultivate industrial hemp for research purposes (though UC established no such program). In June of last year, the FDA approved a CBD-based medicine for treatment of epilepsy-related seizures. With last December's passage of the 2018 Farm Bill, industrial hemp became

\section{UC researchers are vigorously investigating the environmental consequences of cannabis cultivation.}

a legal crop - pending

establishment of a regulatory framework to govern it. Hemp-derived CBD now appears on course for complete de-scheduling by the DEA, and the FDA is wrestling with how to regulate the CBD-based consumer products already hitting the market in many states. Amid this liberalization of federal law on hemp and CBD, it becomes easy to envision UC academics and UCCE personnel performing agronomic studies with hemp - and providing California hemp growers with the same sort of research-based knowledge that has long been available to cultivators of almonds, grapes and lettuce. CA 